http://jmscr.igmpublication.org/home/

ISSN (e)-2347-176x ISSN (p) 2455-0450

crossref DOI: https://dx.doi.org/10.18535/jmscr/v7i12.141

Journal Of Medical Science And Clinical Research

\title{
An out of the Ordinary Case of Delusional Disorder
}

\author{
Authors \\ Dr Ashwathi.J ${ }^{1}$, Dr Vinod G Kulkarni ${ }^{2}$, Dr Mruthyunjaya. ${ }^{3}$, Dr Shashidhara.H.L ${ }^{4}$ \\ ${ }^{1}$ Junior Resident, Department of Psychiatry, SS Institute of Medical Sciences and Research Centre, \\ Davangere \\ ${ }^{2}$ Professor \& HOD, Department of Psychiatry, SSIMS \\ ${ }^{3}$ Assistant Professor, Department of Psychiatry, SSIMS \\ ${ }^{4}$ Associate Professor, Department of Psychiatry, SSIMS
}

\begin{abstract}
Introduction: Delusional disorder is characterised by the presence of non-bizarre, sometimes bizarre delusions of a single theme, in the absence of other mood or psychotic symptoms. We report a patient presenting with somatic symptoms with delusional explanations for the same.

Case History: A 38-yearold male with complaints of chest pain, anxiety and insomnia since 15 days with multiple consultations and investigations for the same was referred to us. History of death of his child 5 months back, following which he became increasingly anxious about his health. Meanwhile, he spotted 3 vermilion stained lemons inside his home following which he firmly believed that his cousins are doing Blackmagic to kill him and to make his reports normal. Though he visited sorcerers, his suspicion continued. Concurrently, when his wife was not well, he convinced by giving the same explanation for her ill-being.

MSE revealed a single, non-bizarre, systematized delusion with partial insight.

Diagnosis and Management: Delusional disorder was diagnosed and started on T. Risperidone $2 m g$ HS and T. Trihexyphenidyl $2 \mathrm{mg} O D$ and was asked to follow up in 15 days.

Discussion: The diagnosis of grief, hypochondriasis and depression were considered. The interesting point here is the delusional explanation for his symptoms for which he has been consulting several doctors which suggests partial insight. Patients with delusional disorder absorb only selectively available information and make conclusions based on less information than healthy subjects and without considering alternative explanations. And therefore, it is difficult to develop therapeutic alliance and patients are likely to refuse medication because they can easily incorporate the administration of drugs into their delusional systems.

Conclusion: Isolated delusions with somatic pain symptoms are rare. However, as with any other delusional disorder, the treatment is challenging in this case because of his partial insight for somatic symptoms and involves both psychopharmacology and psychotherapy. Given the chronic nature of this condition, treatment strategies should be adjusted to the individual needs of the patients with a focus on relieving his anxiety regarding his health problems and improvising his quality of life.

Keywords: Delusional disorder; somatic symptoms.
\end{abstract}

\section{Introduction}

Delusional disorder, an uncommon psychiatric disorder is characterised by the presence of non- bizarre, sometimes bizarre delusions of a single theme, in the absence of other mood or psychotic symptoms ${ }^{1}$. Apart from the direct impact of the 
delusions, impairments in psychosocial functioning may be more circumscribed than those seen in other psychotic disorders such as schizophrenia and behaviour is not obviously bizarre or odd ${ }^{1}$. The prevalence rate is reported to be about $1 \%$ in a psychiatric clinic population in Indian literature ${ }^{2}$.

The disorder is divided into erotomaniac, grandiose, jealous, persecutory and somatic subtypes based on the content of the delusion in which the most common being persecutory subtype in Western literature and delusional parasitosis in Indian literature ${ }^{3}$.We report a case of a middle-aged male who presented with delusional explanation for his somatic symptoms.

\section{Case Report}

A 38 year-old male from a rural background belonging to lower middle socio-economic status, Hindu by religion, educated up to second preuniversity, who is married and owns a general provision store had come with a relative to the outpatient department. He reported that 8 months back, his brother-in-law died due to liver carcinoma following which he started becoming anxious and worried about the functioning of his liver and underwent various investigations and felt relieved after the reports were reported to be normal.

Five months ago, his 15 days old baby passed away as a result of kidney failure after which he started to become increasingly anxious about his health and visited various doctors for complaints of abdominal pain and neck pain. Also underwent many blood and radiological investigations and was not relieved even after the reports were reported to be normal

Around the same time, he spotted three vermilion stained lemons inside his home on distinct days after which he started to firmly believe that his cousins are doing black magic on him to illegally inherit his property by killing him. Also, he believed that they did black magic whenever he was in clinic so that, all his reports and symptoms will be normal. Secondary to these beliefs, he visited several local sorcerers and spent huge amount of money to get rid of the effects that he believed the black magic has caused despite which his fear and suspicion continued. Concurrently, his wife complained of knee joint pain which did not subside even after consulting several doctors. However, he attributed this to black magic and tried to convince her by giving the same explanation.

He complained of chest pain since 15 days and consulted doctors, underwent investigations and was prescribed medications. Although his investigation reports were normal, his fearfulness was persistent. Also, he complained of delayed onset sleep, decreased interest in day-to-day activities and sex, and increased lethargy since the same time. No other unusual beliefs or abnormalities of perception or obsessivecompulsive symptoms was reported. No history of seizures, other organic illness or substance abuse. No family history of any psychiatric illness.

General examination revealed no abnormalities. Blood investigation reports were within normal limits. Mental status examination revealed a fixed belief that his problems were because of the black magic done by his relatives which persisted despite evidence to the contrary suggestive of delusion of persecution. He was alert and oriented with no cognitive deficits and partial insight.

A diagnosis of delusional disorder was considered in view of the single delusional theme in the absence of other psychotic or mood symptoms.

The management focused on establishing rapport with the patient along with eliciting and understanding the explanation he had for his beliefs. Supportive therapy was provided to reduce anxiety and reassure the patient. He was started on antipsychotic medication - risperidone $2 \mathrm{mg}$ one tablet in the night and central anticholinergic- trihexyphenidyl $2 \mathrm{mg}$ one in the morning along with a benzodiazepineclonazepam $0.5 \mathrm{mg}$ one tablet in the night and was explained about the possible adverse effects of the medications and to follow up after 10 days. 


\section{Discussion}

The subject although presented with several somatic symptoms did not have any preoccupation with or fear of having any serious, progressive or life-threatening diseases. Of note is that the patient's personality and psychosocial functioning were not significantly affected by the disorder which helps in ruling out the possibility of hypochondriasis. Also, he has had a stressor in the form of death of his child and his brother. His predominant mood was euthymic with mild anxiety regarding his symptoms and denied any preoccupation with the thoughts of the deceased or any death wishes which rules out grief and depression.

The subject presented with multiple somatic complaints with a delusional explanation for the same. The interesting point in this case is that although he had a delusional explanation for his symptoms he has been consulting several doctors for the same which suggests partial insight. Also, our subject had no family history of delusional disorder. It is sometimes very difficult to distinguish cases of delusional disorder of somatic subtype from severe somatization disorder, and claims have been made that there is a continuum between these illnesses ${ }^{4}$.

The precise aetiology of delusional disorder has not been fully explicated. However, the roles of genetic, biological (hyperdopaminergic states) and psychological factors have been considered ${ }^{5}$. Also, patients with delusional disorder absorb only selectively available information and make conclusions based on less information than healthy subjects and without considering alternative explanations ${ }^{6}$. Difficulties in interpreting others' intentions and motivations seem also to be very common ${ }^{7}$.There are difficulties in developing a therapeutic alliance and patients are likely to refuse medication because they can easily incorporate the administration of drugs into their delusional systems ${ }^{8}$.

\section{Conclusion}

Isolated delusions with somatic pain symptoms are rare. As with any other delusional disorder, the treatment is challenging in this case because of his partial insight for symptoms and involves both psychopharmacology and psychotherapy. Given the chronic nature of this condition, treatment strategies should be adjusted to the individual needs of the patients with a focus on relieving his anxiety regarding his health problems and improvising his quality of life.

\section{References}

1. American Psychiatric Association: Diagnostic and Statistical Manual of Mental Disorders, Fifth Edition. Arlington, VA, American Psychiatric Association, 2013.

2. Hebbar S, Ahuja N, Chandrasekaran R. High prevalence of delusional parasitosis in an Indian setting. Indian J Psychiatry. 1999;41(2):136-9.

3. Noel J, Krishnadas R, Gopalakrishnan R, Kuruvilla A. Delusional Disorder: An Unusual Presentation. Indian J Psychol Med. 2014;36(4):444-6.

4. McElroy SL, Phillips KA, Keck PE, Hudson JI, Pope HG. Body dysmorphic disorder: does it have a psychotic subtype? J Clin Psychiatry. 1993 Oct;54(10):38995.

5. Morimoto K. Delusional Disorder Molecular Genetic Evidence for Dopamine Psychosis. Neuropsychopharmacology. 2002 Jun;26(6):794-801.

6. Altable CR, Castro CV, Santín JML. Concept of Representation and Mental Symptoms. Psychopathology. 2009;42 (4):219-28.

7. Conway CR, Bollini AM, Graham BG, Keefe RSE, Schiffman SS, McEvoy JP. Sensory acuity and reasoning in delusional disorder. Compr Psychiatry. 2002 Jun;43(3):175-8. 
8. Christ WR, Clarkin JF, Hull JW. A highrisk screen for psychiatric discharge planning. Health Soc Work. 1994 Nov;19(4):261-70. 